\title{
Improvements in the Degree of Understanding the Treatment Guidelines for Schizophrenia and Major Depressive Disorder in a Nationwide Dissemination and Implementation Study
}

\section{Shusuke Numata}

Department of Psychiatry, Institute of Biomedical Science, Tokushima University Graduate School

\section{Masahito Nakataki}

Department of Psychiatry, Institute of Biomedical Science, Tokushima University Graduate School Naomi Hasegawa

Department of Pathology of Mental Disease, National Institute of Mental Health, National Center of Neurology and Psychiatry

\section{Yoshikazu Takaesu}

Department of Neuropsychiatry, Kyorin University, Schoool of Medicine

\section{Masahiro Takeshima}

Department of Neuropsychiatry, Akita University Graduate School of Medicine

\section{Toshiaki Onitsuka}

Department of Neuropsychiatry, Graduate School of Medical Sciences, Kyushu University

\section{Toshinori Nakamura}

Department of Psychiatry, Shinshu University School of Medicine

\section{Reon Edagawa}

Department of Psychiatry, Institute of Biomedical Science, Tokushima University Graduate School Hiroaki Edo

Department of Psychiatry, Institute of Biomedical Science, Tokushima University Graduate School

\section{Kenichiro Miura}

Department of Pathology of Mental Disease, National Institute of Mental Health, National Center of Neurology and Psychiatry

\section{Junya Matsumoto}

Department of Pathology of Mental Disease, National Institute of Mental Health, National Center of Neurology and Psychiatry

\section{Norio Yasui-Furukori}

Department of Psychiatry, Dokkyo Medical University, School of Medicine

\section{Taishiro Kishimoto}

Department of Neuropsychiatry, Keio University School of Medicine

\section{Hikaru Hori}


Department of Psychiatry, School of Medicine, University of Occupational and Environmental Health

\section{Takashi Tsuboi}

Department of Neuropsychiatry, Kyorin University

\section{Yuka Yasuda}

Medical Corporation Foster

\section{Ryuji Furihata}

Department of Psychiatry, Nihon University School of Medicine

\section{Hiroyuki Muraoka}

Department of Psychiatry, Tokyo Women`s Medical University

\section{Shinichiro Ochi}

Department of Neuropsychiatry, Molecules and Function, Ehime University Graduate School of Medicine

\section{Tatsuya Nagasawa}

Department of NeuroPsychiatry, Kanazawa Medical University

\section{Toshitaka Kyou}

Department of Psychiatry, Kitasato University, School of Medicine

\section{Atsunobu Murata}

Department of Pathology of Mental Disease, National Institute of Mnetal Health, National Center of Neurology and Psychiatry

\section{Eiichi Katsumoto}

Katsumoto Mental Clinic

\section{Kazutaka Ohi}

Department of Psychiatry and Psychitherapy, Gifu University Graduate School of Medicine

\section{Akitoyo Hishimoto}

Department of Psychiatry, Yokohama City University Graduate School of Medicine

\section{Ken Inada}

Department of Psychiatry, Tokyo Women`s Medical University

\section{Koichiro Watanabe}

Department of Neuropsychiatry, Kyorin University, School of Medicine

\section{Ryota Hashimoto ( $\square$ ryotahashimoto55@ncnp.go.jp )}

Department of Pathology of Mental Diseases, National Institute of Mental Health, National Center of Neurology and Psychiatry, Tokyo, Japan https://orcid.org/0000-0002-5941-4238

\section{Research}

Keywords: dissemination, implementation, clinical practice guidelines, schizophrenia, depression, education, degree of understanding

Posted Date: August 14th, 2020

DOI: https://doi.org/10.21203/rs.3.rs-56435/v1 
License: (c) (i) This work is licensed under a Creative Commons Attribution 4.0 International License. Read Full License

Version of Record: A version of this preprint was published at Neuropsychopharmacology Reports on March 11th, 2021. See the published version at https://doi.org/10.1002/npr2.12173. 


\section{Abstract}

Background: To implement clinical practice guidelines (CPGs), it is necessary for psychiatrists to deepen their understanding of the CPGs. The Effectiveness of Guidelines for Dissemination and Education in Psychiatric Treatment (EGUIDE) project is a nationwide dissemination and implementation study of two sets of CPGs for schizophrenia and major depressive disorder (MDD).

Methods: A total of 413 psychiatrists ( $n=212$ in 2016; $n=201$ in 2017) learned the two CPGs in the education program of the EGUIDE project over two days, and clinical knowledge of the two CPGs was evaluated by two 37-item questionnaires at baseline and after the EGUIDE programs. To improve the correct answer rate for clinical knowledge after the EGUIDE programs, we revised the lecture materials associated with items that had a low correct answer rate in 2016 and used the revised lecture materials with the CPGs in 2017. The rates of correct answers after the EGUIDE programs for each item between the 2016 and 2017 groups were compared.

Results: The total clinical knowledge scores after the EGUIDE program significantly increased in both the 2016 groups and the 2017 groups. We revised lecture materials related to two items in the schizophrenia CPG and five items in the MDD CPG that had low correct answer rates after the EGUIDE programs. The correct answer rate of one item on the schizophrenia CPG and one item on the MDD CPG tended to be improved (S-D5, $p=0.038 ; D-C 6, p=0.033$ ) and that of one on the MDD CPG was significantly improved (D$D 3, p=0.00080$ ) in the 2017 group compared to those in the 2016 group. The other four items were not significantly improved.

Conclusions: We reported improvements in clinical knowledge of CPGs after the EGUIDE program in the 2017 group following revision of the lecture materials based on results from the 2016 group. These attempts to improve the degree of understanding of CPGs may facilitate the successful dissemination and implementation of psychiatric guidelines in everyday practice.

Trial registration: Effectiveness of Guideline for Dissemination and Education in Psychiatric Treatment, UMIN000022645, Registered 15 June 2016, https://upload.umin.ac.jp/cgi-open-bin/ctr/ctr.cgi? function=brows\&action=brows\&recptno=R000026044\&type $=$ summary\&language $=\mathrm{J}$

\section{Contributions To The Literature}

Effective implementation and dissemination of CPGs for psychiatry is necessary, as research has shown that CPGs have minimal effects on clinical practice in the context of mental health pharmacotherapy.

The EGUIDE project is a nationwide dissemination and implementation study of CPGs for psychiatry. We revealed that the clinical knowledge of CPGs improved by the EGUIDE program. We also provided evidence for the effectiveness of lecture revisions, which further improved the clinical knowledge of the CPGs. 
This attempt to improve the clinical knowledge of CPGs can facilitate the successful dissemination and implementation of psychiatric guidelines in everyday practice.

\section{Background}

Clinical practice guidelines (CPGs) include recommendations intended to optimize patient care that are informed by a systematic review of evidence as well as an assessment of the benefits and harms of alternative care [1]. Schizophrenia and major depressive disorder (MDD) are major psychiatric disorders, and international CPGs for these two disorders, such as those from the American Psychiatric Association (APA) [2] and the National Institute for Health and Clinical Excellence (NICE) [3], have been published in multiple countries. In Japan, the "Guideline for Pharmacological Therapy for Schizophrenia" (CPG for schizophrenia) was developed by the Japanese Society of Neuropsychopharmacology in 2015 [4], and the "Treatment Guideline II: Major Depressive Disorder" (CPG for MDD) was published by the Japanese Society of Mood Disorders in 2016 [5]. On the other hand, CPG dissemination and implementation strategies for psychiatric disorders have not been established to date [6]. To disseminate and implement these two CPGs, the Effectiveness of Guidelines for Dissemination and Education in Psychiatric Treatment (EGUIDE) project was started in $2016[7,8]$. The EGUIDE project started with the cooperation of 22 hospitals, and as of 2020, more than 180 hospitals (44 universities) participated. In brief, education programs for psychiatrists regarding these two CPGs are provided throughout Japan. The efficacy of the EGUIDE project is evaluated using three quality indicators: the degree of understanding the guidelines, the degree of practice, and the prescribing behavior of each psychiatrist. The programs are reviewed every year based on the previous year's resultsto improve the degree of understanding of the guidelines. The final goal of the EGUIDE project is the successful implementation of psychiatric CPGs in everyday practice. Recently, we reported that clinical knowledge of the guidelines was significantly improved after participation in the education programs [7]. In the current study, we evaluated the effect of revision of the lecture materials on the knowledge of the guidelines after attending the educational program by comparing participant data from two years.

\section{Methods}

The EGUIDE projecthas two sets of implementation content, the development of the CPGs and lectures associated with the CPGs, and three quality indicators, clinical knowledge, clinical behavior, and prescribing behavior $[7,8]$. This study aimed to examine the effect of revision of the lecture materials on improvements in clinical knowledge in 2017 based on the feedback loop from evaluation of clinical knowledge following lectures on the CPGs (Figure 1).

\section{Participants}

From October 2016 to March 2017, 212 psychiatrists participated in both the CPG for the schizophreniaprogram and the CPG for the MDDprogram. From October 2017 to March 2018, a new group of 201 psychiatrists took these two programs [7]. 


\section{Assessment of clinical knowledge of schizophrenia and MDD CPGs}

Clinical knowledge of the two CPGs was evaluated using a questionnaire at baseline and after the EGUIDE programs. Assessment measures on the questionnaire have been previously described [7]. Just before the class started and after the class ended, the participants completed a self-administered questionnaire that assessed their knowledge of the clinical guidelines. The questionnaire for the CPG for schizophrenia consisted of seven parts (for a total of 37 questions), and the CPG for MDD consisted of eight parts (a total of 37 questions).

\section{Revision of the lecture materials}

The correct answer rates regarding clinical knowledge at baseline and after the EGUIDE program for the CPG for schizophrenia in the 2016 group are shown in Additional file 1, and those for the 2017 group are shown in Additional file 2. The correct answer rate regarding clinical knowledge at baseline and after the EGUIDE program for the CPG for MDD in the 2016 group are shown in Additional file 3, and those for the 2017 group are shown in Additional file 4.

In response to the low correct answer rate regarding clinical knowledge after the EGUIDE programs, we decided to revise the lecture materials for the purpose of increasing the correct answer rates. Therefore, we defined three categories based on the correct answer rates for the clinical knowledge items (category A: correct answer rates at baseline and after the program were over $90 \%$; category B: correct answer rates were less than $90 \%$ at baseline and over $90 \%$ after the program; category C: correct answer rates at baseline and after the program were less than $90 \%$ ). To examine improvements in the correct answer rates regarding clinical knowledge after the EGUIDE programs, we revised lecture materials related to the items in category C in 2016 and used the revised material in the lectures for the CPGs in 2017.

\section{Statistical analysis}

Statistical analyses were performed using R software (ver. 4.0.0) with "exactRankTests" and "moments" packages. The normality of the clinical knowledge scores and participant age and professional experience were evaluated using the omnibus K-squared test, Shapiro-Wilk test, and Kolmogorov-Smirnov test. The demographic differences between the two groups in age and professional experience were compared using Wilcoxon rank-sum tests, and differences in gender were compared using Fisher's exact test. The total clinical knowledge scores at baseline and after the EGUIDE program were compared using the Wilcoxon signed-rank tests in the 2016 and 2017 groups. The correct answer rates after the EGUIDE program for each item in the 2016 and 2017 groups were compared using Fisher's exact tests. The level of statistical significance was set at $p<0.05$.

\section{Results}

\section{Characteristics of participants}


Of the 212 participants from October 2016 to March 2017, data for 168 participants (2016 group) were available. Of the 201 participants from October 2017 to March 2018, data for 176 (2017 group) were available. The demographic characteristics of each group are presented in Table 1. The mean age was $33.9 \pm 6.7$ years (mean \pm standard deviation) for the 2016 group and $33.7 \pm 7.1$ years for the 2017 group. The mean years of professional experience for each group was $4.9 \pm 5.5$ and $5.3 \pm 6.6$ years, respectively, and the male:female ratio of each group was 2.29 and 3.76, respectively. There were no significant differences between the 2016 and 2017 groups in age or years of professional experience $(p=0.52$ and $p$ $=0.15$, respectively). However, there were significant differences in the gender ratio between the two groups $(p=0.049)$.

\section{Clinical knowledge after the EGUIDE program in 2016}

In the 2016 group, the total clinical knowledge scores on the CPG for schizophrenia at baseline and after the EGUIDE program were $33.6 \pm 2.2$ and $36.2 \pm 1.0$ points $\left(p=1.4 \times 10^{-25}\right)$. Although total scores improved after the program, the correct answer rates after the EGUIDE program of two questions did not reach over $90 \%$, and these questions were classified as category 3 items: S-D5 and S-F4 $(72.6 \%$ and $83.3 \%$, respectively; Figure $2 \mathrm{~A}$, Additional file 1). The total clinical knowledge scores on the CPG for MDD at baseline and after the program were $31.0 \pm 2.7$ and $34.4 \pm 1.8$ points $\left(p=4.8 \times 10^{-27}\right)$. Although total scores improved after the program, the correct answer rates after the EGUIDE program on five questions did not reach over $90 \%$, and these questions were classified as category 3 items: D-A1, D-B5, D-C5, D-C6 and D-D3 (56.5\%, 47.6\%, 71.4\%, 85.7\%, 78.6\%, respectively; Figure 2B, Additional file 3).

\section{Improvement of lectures for 2017}

Based on the results of the 2016 group after the EGUIDE programs, lecture materials were revised for the 2017 group, with particular attention paid to questions with low correct answer rates: two questions for the schizophrenia CPG and five questions for the MDD CPG.

For S-D5, an additional slide was created that emphasized not combining psychotropics such as mood stabilizers, anxiolytics, and antidepressants with antipsychotics in the lecture on the management of recurrence or relapse in schizophrenia. For S-F4, emphasis was placed on the idea that modified electroconvulsive therapy (ECT) may be effective in patients with treatment-resistant schizophrenia when clozapine is not available at the end of the lecture on the management of treatment-resistant schizophrenia. For D-A1, the need to accurately confirm the diagnostic criteria was emphasized in the lecture on the diagnosis of MDD according to the Diagnostic and Statistical Manual of Mental Disorders (DSM-5). For D-B5, an additional slide was created, which emphasized that adverse effects of antidepressants should be carefully considered with not only children and elderly patients with MDD but also pregnant patients with MDD in the lecture on treatment of MDD. For D-C5, it was emphasized that patient preference should be prioritized (e.g., antidepressant use is not always necessary) in the management of mild depression. For D-C6, the notion that antidepressants may be effective in patients with prior good treatment response to antidepressants and/or multiple depressive episodes was added to 
the lecture materials. For D-D3, in the lecture on moderate/severe depression, an additional slide was created, which emphasized that, if necessary, benzodiazepines can be used for a short period of time.

\section{Effects of revised lecture materials on clinical knowledge after the EGUIDE program in 2017}

In the 2017 group, the total clinical knowledge scores at baseline and after the EGUIDE program with the CPG for schizophrenia were $33.2 \pm 2.5$ and $36.4 \pm 0.8$ points, respectively $\left(p=4.0 \times 10^{-27}\right)$.To examine the effect of the revised lecture materials on the correct answer rates after the EGUIDE program with the CPG for schizophrenia, we compared of the correct answer rates for the two category $C$ items between the 2016 group and the 2017 group (S-D 5: $72.6 \%$ in 2016, 82.4\% in 2017, $p=0.038$; S-F4: $83.3 \%$ in 2016, $86.4 \%$ in $2017, p=0.45 ;$ Table 2). The improvement on S-D5 did not reach statistical significance when corrected for multiple testing (Bonferroni correction, $\mathrm{p}<0.025$ ).

Similarly, the total clinical knowledge scores at baseline and after the EGUIDE program with the CPG for MDDwere $31.4 \pm 2.6$ and $34.8 \pm 1.6$ points, respectively $\left(p=2.3 \times 10^{-27}\right)$.To examine the effect of the revised lecture materials on the correct answer rates after the EGUIDE program with the CPG for MDD, we compared the correct answer rates on the five category C items between the 2016 group and the 2017 group (D-A1: $56.5 \%$ in 2016, 60.8\% in 2017, $p=0.44$; D-B5: $47.6 \%$ in $2016,51.1 \%$ in 2017 , p = 0.52; D-C5: $71.4 \%$ in $2016,65.9 \%$ in 2017, $p=0.30$; D-C6: $85.7 \%$ in 2016, 93.2\% in 2017, $p=0.033$; D-D3: $78.6 \%$ in $2016,89.2 \%$ in 2017, $p=8.0 \times 10-3$; Table 3). The difference in the correct answer rate on D-C6 after the EGUIDE program did not reach statistical significance when corrected for multiple testing, but the rate exceeded $90 \%$. The correct answer rates on D-D3 after the EGUIDE program were significantly improved and rose to approximately $90 \%$.

\section{Discussion}

The aim of the EGUIDE project is the successful implementation of CPGs for psychiatrists in everyday practice. This study evaluated clinical knowledge after revision of lecture materials in the context of a feedback loop. As a result, the total clinical knowledge scores after the EGUIDE program significantly increased in both the 2016 and 2017 groups. Among the category C items in 2016, the revised lecture materials sufficiently affected item D-D3, which was a question about the combination of antidepressants and benzodiazepines or atypical antipsychotics in the treatment for moderate/severe depression. Our findings suggest that revising lecture materials improved clinical knowledge.

The EGUIDE project is a nationwide dissemination and implementation study for the CPGs for schizophrenia and MDD. We collected outcomes on participants in three quality indicators: the degree of understanding the guidelines, the degree of practice, and the prescribing behavior. The EGUIDE project has multiple dissemination methods and evaluation methods, each of which is improved by feedback loops and progresses step by step. To our knowledge, this is the first study to provide dissemination and implementation and to evaluate multiple quality indicators of CPGs in the psychiatric field. 
In a meta-analysis examining the impact of guidelines on clinical practice in psychiatry, including psychosis and MDD, Nguyen and colleagues found that CPGs frequently had minimal or insubstantial effects on clinical practice in the context of mental health pharmacotherapy [9]. In Japan, prescribing behaviors that did not follow CPGs have been repeatedly reported in the treatment of schizophrenia and MDD [10-16]. Taken together, these results suggest that it is not easy for psychiatrists to prescribe according to the CPGs and that additional trials may be needed for the successful implementation of CPGs in clinical practice. Personal factors, such as lack of awareness and/or familiarity with guidelines, have been determined to be a major factor in the barrier to CPG implementation $[17,18]$. Therefore, the supply of proper educational materials is essential to raise awareness of and increase familiarity with the CPGs [18]. Attempts to improve the degree of understanding of the treatment guidelines by revising lecture materials of educational programs each year will help to facilitate the successful implementation of psychiatric guidelines to everyday practice because knowledge influences attitudes and attitudes impact practice behavior. Item D-D3, which showed an improvement among the clinical knowledge items, was a question regarding the combination therapy of benzodiazepines and antipsychotics with antidepressants for MDD. In Japan, polypharmacy with benzodiazepines for MDD is a serious problem [19], so it is very meaningful to improve the correct answer rate on a clinical knowledge item that does not recommend this. Our aim is to achieve optimized treatment in psychiatry, and further studies to investigate prescription patterns after EGUIDE programs will be necessary.

There are several limitations in the current study. The participants were recruited from various facilities across Japan, and they had not received common training at each facility prior to attending the EGUIDE project, although there were no significant differences in age or years of professional experience between the 2016 and 2017 groups. Our sample size was small, limiting the ability to generalize the results to the approximately 14,000 psychiatrists in Japan. Correlations between the degree of understanding the guidelines and the degree of practice and prescribing behavior in the present study were not shown since the EGUIDE project was started in 2016 and is still in progress. There were still several questions with low correct answer rates in the 2017 group after the EGUIDE programs, suggesting that further revisions of the lecture materials will be needed. It is possible that the effective revision of the lecture materials was the addition of slides. We considered that other causes of low correct answer rates after the EGUIDE program were that the training materials were difficult to understand, the questions on the clinical knowledge test are not suitable and the imprint of past lectures prevented interference with the EGUIDE program. Based on the above, we will continue to discuss the opinions from the participants and the instructing psychiatrist of the EGUIDE project and consider improvements.

\section{Conclusions}

In conclusion, we reported improvements in clinical knowledge of the CPGs after the EGUIDE program in the 2017 group by revision of the lecture materials based on the results from the 2016 group. Achieving a good understanding of psychiatric treatment guidelines is an important step in raising awareness and increasing familiarity with these guidelines. The attempts to improve the degree of understanding of the 
treatment guidelines may facilitate the successful dissemination and implementation of psychiatric guidelines to everyday practice in the future.

\section{Abbreviations}

CPGs: Clinical Practice Guidelines; MDD: major depressive disorder; APA: American Psychiatric Association; NICE: National Institute for Health and Clinical Excellence; EGUIDE: Effectiveness of Guidelines for Dissemination and Education in Psychiatric Treatment

\section{Declarations}

\section{Acknowledgments}

We appreciate the cooperation of all the individuals who participated in this study.

\section{Author contributions}

$\mathrm{SN}$ and $\mathrm{NH}$ were involved in data collection and data analysis and wrote the first draft of the manuscript. $M N, R E, H E, Y T, M T, T O, T N, K M$ and JM were involved in the data analysis and contributed to the interpretation of the data and writing of the manuscript. NY-F, TK, HH, TT, YY, RF, HM, SO, TN, YK, AM, EK, $\mathrm{KO}$, and $\mathrm{AH}$ contributed to the interpretation of the data and data collection. $\mathrm{KW}$ and $\mathrm{KI}$ were involved in the study design and contributed to the interpretation of the data. RH supervised the entire project, collected the data and was involved in the design, analysis, and interpretation of the data. All authors contributed to and approved the final article.

\section{Funding}

This study was supported by the Japan Agency for Medical Research and Development (AMED) under Grant Number JP 18dk0307060, the AMED under Grant Number JP 19dk0307083, the Health and Labor Science Research Grants (H29-Seishin-Ippan-001, 19GC1201), the Japanese Society of Neuropsychopharmacology and the Japanese Society of Mood Disorders. The funders had no role in the study design, data collection and analyses, decision to publish, or preparation of the manuscript.

\section{Availability of data and materials}

The dataset (which includes individual transcripts) is not publicly available due to confidentiality policies.

\section{Ethics approval and consent to participate}

This study was conducted in accordance with the Declaration of Helsinki. The protocol of this study was registered in the University Hospital Medical Information Network registry (UMIN000022645). This study was approved by the ethics committees of the National Center of Neurology and Psychiatry (A2017-105) 
and each participating university, hospital and clinic. All participants provided their written informed consent.

\section{Consent for publication}

Not applicable

\section{Competing interests}

The authors declare that they have no competing interests.

\section{References}

1. Institute of Medicine (US) Committee on Standards for Developing Trustworthy ClinicalPractice Guidelines; Graham R, Mancher M, Miller Wolman D, Greenfield S, Steinberg E, editors. ClinicalPractice Guidelines We Can Trust. Washington (DC): NationalAcademiesPress (US); 2011.

2. American Psychiatric Association (APA). Practice Guideline for the Treatment of Patients with Major Depressive Disorder (third ed.). 2011

3. National Institute for Health and Clinical Excellence (NICE). The treatment and management of depression in adults (updated edition)-National Clinical Practice Guideline. 2010.

4. Japanese Society of Neuropsychopharmacology. Guideline for Pharmacological Therapy for Schizophrenia. Igakusyoin, Tokyo. 2015 (in Japanese).

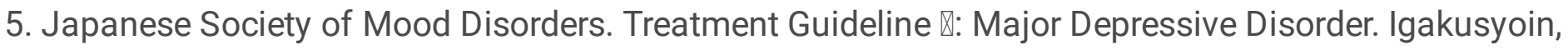
Tokyo. 2016 (in Japanese).

6. Bighelli I, Ostuzzi G, Girlanda F, Cipriani A, Becker T, Koesters M, et al. Implementation of Treatment Guidelines for Specialist Mental Health Care Cochrane Database Syst Rev. 2016;12:CD009780.

7. Takaesu Y, Watanabe K, Numata S, Iwata M, Kudo N, Oishi S, et al. Improvement of psychiatrists' clinical knowledge of the treatment guidelines for schizophrenia and major depressive disorders using the 'Effectiveness of Guidelines for Dissemination and Education in Psychiatric Treatment (EGUIDE)' project: A nationwide dissemination, education, and evaluation study.Psychiatry Clin Neurosci. 2019;73:642-648.

8. Ichihashi K, Hori H, Hasegawa N, Yasuda Y, Yamamoto T, Takashi et al. Prescription patterns in patients with schizophrenia in Japan: first-quality indicator data from the survey of "Effectiveness of Guidelines for Dissemination and Education in psychiatric treatment (EGUIDE)" project. Neuropsychopharmacol Rep. 2020 [in press].

9. Nguyen T, Seiler N, Brown E, O'Donoghue B. The effect of Clinical Practice Guidelines on prescribing practice in mental health: A systematic review.Psychiatry Res. 2020;284:112671.

10. Ito $\mathrm{H}$, Koyama A, Higuchi T. Polypharmacy and excessive dosing: psychiatrists' perceptions of antipsychotic drug prescription.Br J Psychiatry. 2005;187:243-247. 
11. Uchida H, Suzuki T, Mamo DC, Mulsant BH, Tsunoda K, Takeuchi H, et al. Survey of benzodiazepine and antidepressant use in outpatients with mood disorders in Japan.Psychiatry Clin Neurosci. 2009;63:244-246.

12. Onishi Y, Hinotsu S, Furukawa TA, Kawakami K. Psychotropic prescription patterns among patients diagnosed with depressive disorder based on claims database in Japan.Clin Drug Investig. 2013;33:597-605.

13. Nakagawa A, Williams A, Sado M, Oguchi Y, Mischoulon D, Smith F, et al. Comparison of treatment selections by Japanese and US psychiatrists for major depressive disorder: A case vignette study.Psychiatry Clin Neurosci. 2015;69:553-62.

14. Yang SY, Chen LY, Najoan E, Kallivayalil RA, Viboonma K, Jamaluddin R, et al. Polypharmacy and Psychotropic Drug Loading in Patients With Schizophrenia in Asian Countries: Fourth Survey of Research on Asian Prescription Patterns on Antipsychotics. Psychiatry Clin Neurosci. 2018;72:572579.

15. Takahashi T, Otsubo T, Kunisawa S, Noriko S, Imanaka Y. Factors Associated With High-Dose Antipsychotic Prescriptions in Outpatients With Schizophrenia: An Analysis of Claims Data From a Japanese Prefecture. Neuropsychopharmacol Rep. 2020 [Online ahead of print].

16. Usuki M, Kamiura Y, Okubo R, Yamanouchi Y. National Database Study on the Use of Long-Acting Antipsychotic Injections and Hospital Readmission Proportions in Patients With Schizophrenia in Japan. Psychiatry Clin Neurosci. 2020;74:211-217.

17. Cabana MD, Rand CS, Powe NR, Wu AW, Wilson MH, Abboud PA, et al. Why don't physicians follow clinical practice guidelines? A framework for improvement.JAMA. 1999;282:1458-65.

18. Fischer F, Lange K, Klose K, Greiner W, Kraemer A. Barriers and Strategies in Guideline Implementation-A Scoping Review.Healthcare (Basel). 2016;4:pii: E36.

19. Onishi Y, Hinotsu S, Furukawa T A, Kawakami K. Psychotropic prescription patterns among patients diagnosed with depressive disorder based on claims database in Japan. Clin Drug Investig. 2013;33:8:597-605.

\section{Tables}

\section{Table 1. Characteristics of participants}




\begin{tabular}{|c|c|c|c|c|c|c|c|}
\hline & & \multicolumn{3}{|c|}{2016 group $(N=168)$} & \multicolumn{3}{|c|}{2017 group $(N=176)$} \\
\hline & & Male & Female & Total & Male & Female & Total \\
\hline & & $(\mathrm{N})$ & $(\mathrm{N})$ & $(\mathrm{N})$ & $(\mathrm{N})$ & $(\mathrm{N})$ & $(\mathrm{N})$ \\
\hline \multirow[t]{4}{*}{ Age (years) } & $20 \sim 29$ & 30 & 22 & $\begin{array}{l}52 \\
(31.0 \%)\end{array}$ & 40 & 18 & $\begin{array}{l}58 \\
(33.0 \%)\end{array}$ \\
\hline & $30 \sim 39$ & 66 & 22 & $\begin{array}{l}88 \\
(52.4 \%)\end{array}$ & 70 & 16 & $\begin{array}{l}86 \\
(48.9 \%)\end{array}$ \\
\hline & $40 \sim 49$ & 16 & 6 & 22 & 21 & 3 & $\begin{array}{l}24 \\
(13.6 \%)\end{array}$ \\
\hline & $\begin{array}{l}\text { Over } \\
50\end{array}$ & 5 & 1 & $6(3.6 \%)$ & 8 & 0 & $\begin{array}{l}8 \\
(4.5 \%)\end{array}$ \\
\hline \multirow[t]{4}{*}{$\begin{array}{l}\text { Professional experience } \\
\text { (years) }\end{array}$} & $1 \sim 3$ & 68 & 32 & $\begin{array}{l}100 \\
(59.5 \%)\end{array}$ & 74 & 29 & $\begin{array}{l}103 \\
(58.5 \%)\end{array}$ \\
\hline & $4 \sim 6$ & 20 & 12 & 32 (19\%) & 21 & 4 & $\begin{array}{l}25 \\
(14.2 \%)\end{array}$ \\
\hline & 7 9 & 11 & 3 & 14 (8.3\%) & 15 & 1 & $\begin{array}{l}16 \\
(9.1 \%)\end{array}$ \\
\hline & $\begin{array}{l}\text { Over } \\
10\end{array}$ & 18 & 4 & $\begin{array}{l}22 \\
(13.1 \%)\end{array}$ & 29 & 3 & $\begin{array}{l}32 \\
(18.2 \%)\end{array}$ \\
\hline
\end{tabular}

Table 2. Correct answer rates regarding clinical knowledge after the EGUIDE program with the CPG for schizophrenia

\begin{tabular}{|lllll|}
\hline Schizophrenia & Items & $2016(\%)$ & 2017 (\%) & p value \\
\hline Management of recurrence or relapse of schizophrenia & & & & \\
\hline $\begin{array}{l}\text { Do not use psychotropics other than antipsychotics } \\
\text { [correct] }\end{array}$ & S-D5 & 72.6 & 82.4 & 0.038 \\
\hline Management of treatment-resistant schizophrenia & & & & \\
\hline $\begin{array}{l}\text { Modified electroconvulsive therapy is recommended } \\
\text { [correct] }\end{array}$ & S-F4 & 83.3 & 86.4 & 0.45 \\
\hline
\end{tabular}

Table 3. Correct answer rates regarding clinical knowledge after the EGUIDE program with the CPG for major depressive disorder 
2016

(\%)

2017 p value

(\%)

Diagnosis of major depressive disorder (DSM-5)

Depressed mood and a loss of interest or pleasure most of the day, nearly every day should be present over the same two-week

D-A1

56.5

60.8

0.44
Treatment of major depressive disorder

The safety of antidepressants during pregnancy has not been not determined, so more attention to adverse effects should be paid when antidepressants are administered to young people than to the elderly [incorrect]

Management of mild depression

Antidepressants are not recommended when patients expect to receive psychotherapy [correct]

Antidepressant use should be avoided when patients have multiple depressive episodes [incorrect]

Recommended treatment for moderate/severe depression

Combination of antidepressants and benzodiazepines or atypical antipsychotics with adequate doses and duration [incorrect]
D-B5 $\quad 47.6 \quad 51.1 \quad 0.52$

D-C5 $\quad 71.4 \quad 65.9 \quad 0.3$

$\begin{array}{llll}\text { D-C6 } & 85.7 & 93.2 & \mathbf{0 . 0 3 3}\end{array}$

D-D3 $78.6 \quad 89.2 \quad \underline{\mathbf{8 . 0 \times 1 0 ^ { - 3 }}}$

\section{Figures}




\section{Improvement steps}

Three quality indicators

\section{Goal}

Two implementation contents

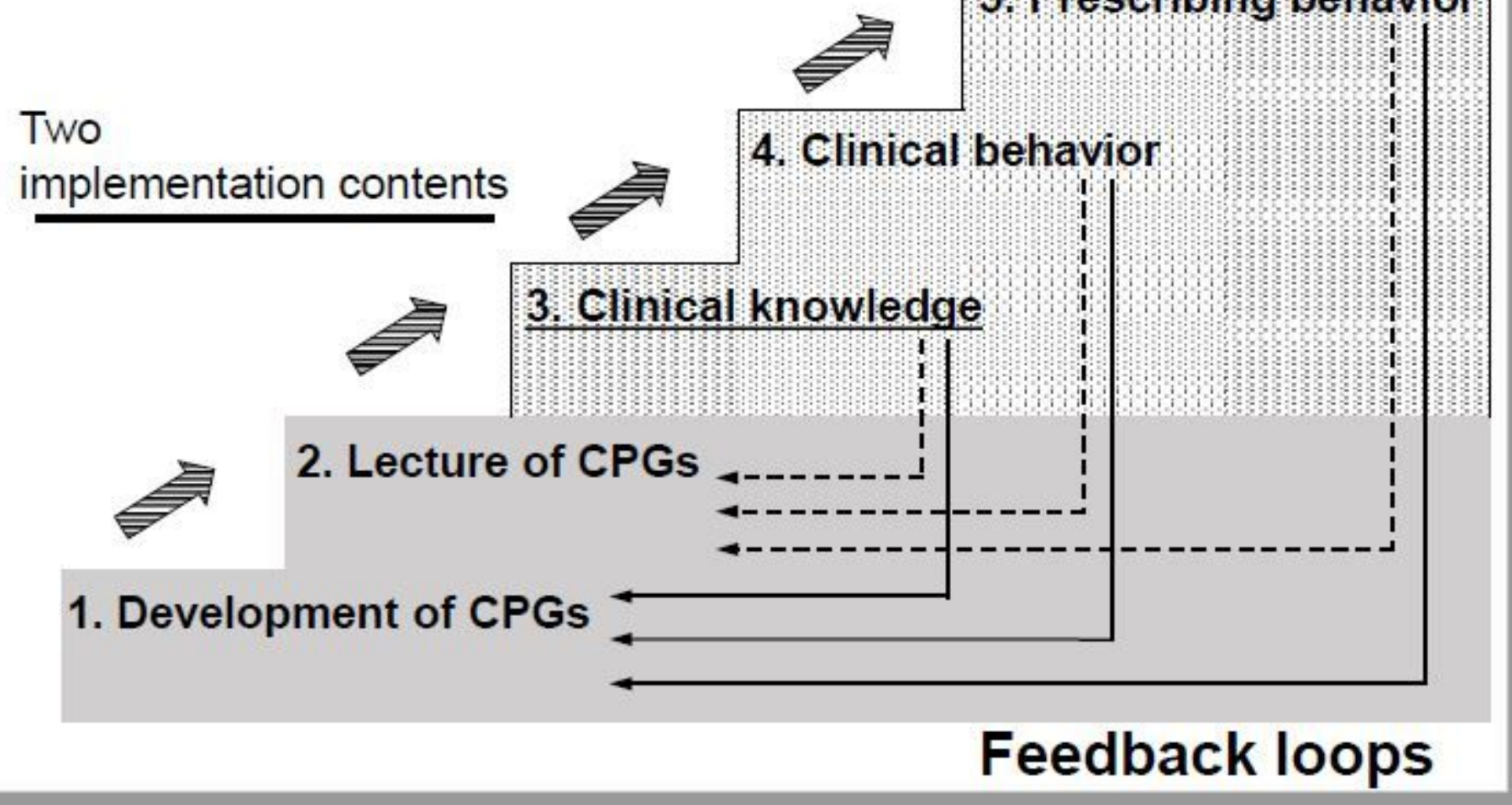

Patients' QOL

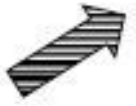

5. Prescribing behavior

Figure 1

The strategy of the EGUIDE project. The EGUIDE project has two sets of implementation content, the development of CPGs and the lectures associated with the CPGs, and three quality indicators, clinical knowledge, clinical behavior, and prescribing behavior. Striped arrows represent the flow of improvement steps. Dotted and solid arrows represent the flow of feedback loops. 
(A)

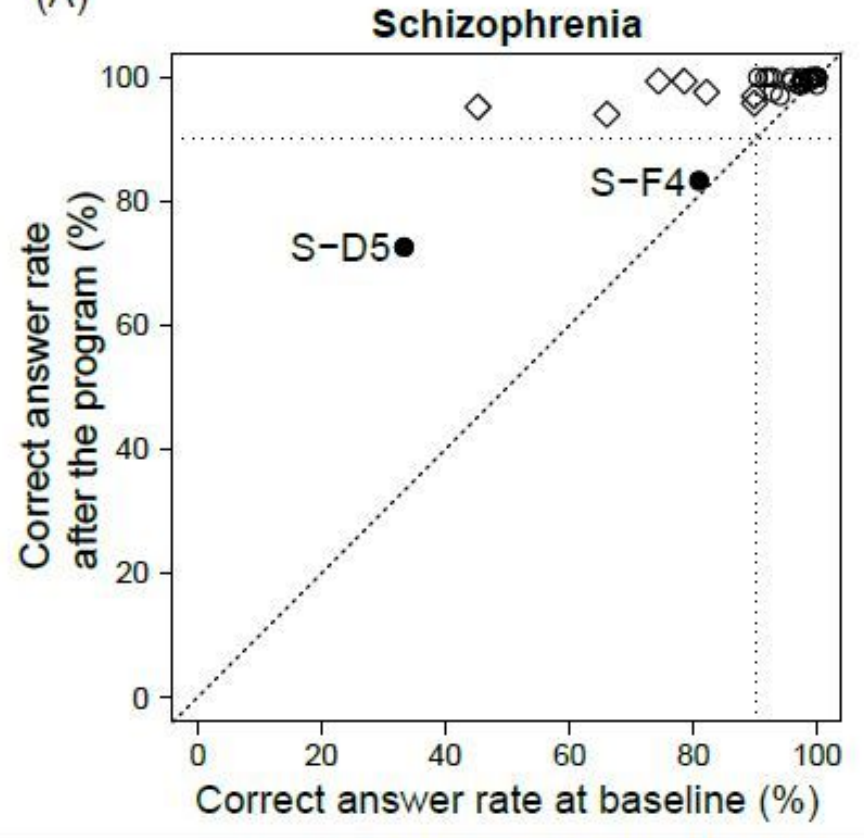

(B)

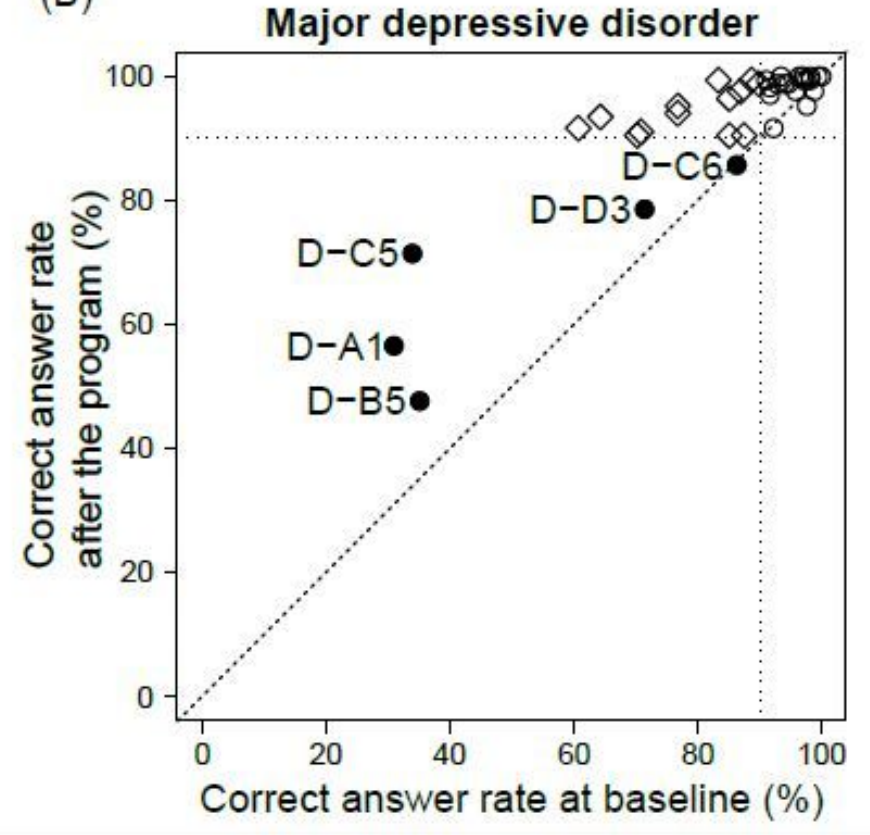

Figure 2

Correct answer rates for each question regarding clinical knowledge in the 2016 group (A) The x-axis represents correct answer rates for each question at baseline. The y-axis represents correct answer rates for each question after the CPG for the schizophrenia program. Category A: correct answer rates were over $90 \%$ at baseline and after the program (S-A1, A2, A3, B1, B2, B3, B4, B5, C1, C2, C3, D1, D2, D3, D4, E1, E4, E6, F1, F2, F3, G2, G3, G4, G5, G6, G7, and S-G8). \ Category B: correct answer rates were less than $90 \%$ at baseline and over $90 \%$ after the program (S-A4, E2, E3, E5, F5, F6, and S-G1). Category C: correct answer rates were less than $90 \%$ at baseline and after the EGUIDE program (S-D5 and S-F4). (B) The $x$ axis represents correct answer rates for each question at baseline. The y-axis represents correct answer rates for each question after the $\mathrm{CPG}$ for the MDD program. Category A: correct answer rates were over $90 \%$ at baseline and after the program (D-A3, A4, A5, B1, B3, B4, C2, C3, C4, D1, E1, E2, E3, G2, G3, H1, H2, $\mathrm{H} 3$, and $\mathrm{D}-\mathrm{H} 4)$. $\triangle$ Category $\mathrm{B}$ : correct answer rates were less than $90 \%$ at baseline and over $90 \%$ after the program (D-A2, A6, B2, C1, C7, D2, D4, F1, F2, F3, F4, G1, and D-G4). OCategory C: correct answer rates were less than $90 \%$ at baseline and after the EGUIDE program (D-A1, B5, C5, C6, and D-D3).

\section{Supplementary Files}

This is a list of supplementary files associated with this preprint. Click to download.

- additionalfileadherencetoreportingguideline.docx

- EGUIDEunderstand2Af406.xIsx

- EGUIDEunderstand2Af307.xIsx 
- EGUIDEunderstand2Af207.xIsx

- EGUIDEunderstand2Af106.xIsx 